\title{
Relationship between the Sunspot Number and Solar Polar Field by Wavelet-Based Multifractal Analysis
}

\author{
Fumio Maruyama \\ Department of Sport and Health Science, Matsumoto University, Matsumoto, Japan \\ Email:fmaruya@nagoya-u.jp
}

How to cite this paper: Maruyama, F. (2019) Relationship between the Sunspot Number and Solar Polar Field by Wavelet-Based Multifractal Analysis. Journal of Applied Mathematics and Physics, 7, 1043-1051.

https://doi.org/10.4236/jamp.2019.75070

Received: April 13, 2019

Accepted: May 14, 2019

Published: May 17, 2019

Copyright (c) 2019 by author(s) and Scientific Research Publishing Inc. This work is licensed under the Creative Commons Attribution International License (CC BY 4.0).

http://creativecommons.org/licenses/by/4.0/

\begin{abstract}
There is increasing interest in finding the relation between the sunspot number (SSN) and solar polar field. In general, fractal properties may be observed in the time series of the dynamics of complex systems, such as solar activity and climate. This study investigated the relations between the SSN and solar polar field by performing a multifractal analysis. To investigate the change in multifractality, we applied a wavelet transform to time series. When the SSN was maximum and minimum, the SSN showed monofractality or weak multifractality. The solar polar field showed weak multifractality when that was maximum and minimum. When the SSN became maximum, the fractality of the SSN changed from multifractality to monofractality. The multifractality of SSN became large before two years of SSN maximum, then that of the solar polar field became large and changed largely. It was found that the change in SSN triggered the change in the solar polar field. Hence, the SSN and solar polar field were closely correlated from the view point of fractals. When the maximum solar polar field before the maximum SSN was larger, the maximum SSN of the next cycle was larger. The formation of the magnetic field of the sunspots was correlated with the solar polar field.
\end{abstract}

\section{Keywords}

Sunspot Number, Solar Polar Field, Wavelet, Multifractal

\section{Introduction}

Various objects in nature exhibit the so-called self-similarity or fractal property. Nature is full of fractals, for instance, trees, rivers, coastlines, mountains, clouds, and seashells. Monofractal signals are homogeneous in that they have the same scaling properties and are characterized by a fractal dimension. On the other 
hand, multifractal signals are nonuniform, more complex and can be decomposed into many subsets characterized by the different dimensions of the fractal. Fractal properties can also be observed in a time series representing the dynamics of complex systems. A change in fractality can appear with a phase transition or change of state. As an example, the multifractal properties of daily rainfall were studied in an East Asian monsoon climate with extreme rainfall and in a temperate climate with moderate rainfall [1]. In both climates, the frontal rainfall and convective-type rainfall showed monofractality and multifractality, respectively. As another example, a healthy human heartbeat shows a multifractal character, while a diseased heart shows a monofractal character [2].

The sunspot number (SSN) has an 11-year cycle and the solar magnetic field has a 22-year cycle, exactly twice that of the sunspot cycle, because the polarity of the field returns to its original value every two sunspot cycles. During the past $\sim 120$ years, Earth's surface temperature has been correlated with both the decadal averages and solar cycle minimum values of the geomagnetic aa index [3]. The 11-year averages of the SSN and aa index have been highly correlated for the past 150 years [4]. The maxima of the aa index occurred near the maximum SSN and (or) later in the declining phase of the SSN [5] [6]. High correlation coefficients were found among the geomagnetic activity, sea level atmospheric pressure and surface air temperature [7].

The aim of our work is to investigate the relations between the SSN and solar polar field from the view point of fractals. To examine the changes in multifractality, we performed a multifractal analysis on the SSN, and solar polar field by using the wavelet transform. The wavelet transform can perform reliable multifractal analysis [8]; hence, we used the wavelet-based multifractal analysis to quantify the signals of higher complexity.

\section{Data and Method of Analysis}

We used several solar activity indices to see the solar activity in a multifaceted manner. We used the monthly SSN provided by Solar Influences Data Analysis Center (sidc.oma.be) and the solar polar field throughout the solar sunspot cycle provided by the Wilcox Solar Observatory (http://wso.stanford.edu/).

For the analysis, we used the Daubechies wavelet, which is widely used in solving a broad range of problems, e.g., self-similarity properties of a signal or fractal problems and signal discontinuities. We used a discrete signal which was fitted the Daubechies mother wavelet with the capacity of accurate inverse transformation. Thus, we can precisely calculate the following optimum $\tau(q)$, which can be regarded as a characteristic function of the fractal behavior. We can define the $\tau(q)$ from the power-law behavior of the partition function, as shown in Equation (2). We then calculated the scaling of the partition function $Z_{q}(a)$, which is defined as the sum of the $q$-th powers of the modulus of the wavelet transform coefficients at scale $a$, where $q$ is the $q$-th moment. In our calculation, the wavelet-transform coefficients did not become zero. Thus, for an 
accurate calculation, the summation was considered for the whole set. Muzy et al. [8] defined $Z_{q}(a)$ as the sum of the $q$-th powers of the local maxima of the modulus to avoid dividing by zero. We obtained the following partition function $Z_{q}(a)$ :

$$
Z_{q}(a)=\sum\left|W_{\varphi}[f](a, b)\right|^{q},
$$

where $W_{\varphi}[f](a, b), a$, and $b$ are the wavelet coefficient of function $f$, a scale parameter, and a space parameter, respectively. $W_{\phi}[f](a, b)$ is defined as below.

$$
W_{\phi}[f](a, b)=\frac{1}{\sqrt{|a|}} \int_{-\infty}^{+\infty} f(t) \varphi^{*}\left(\frac{t-b}{a}\right) \mathrm{d} t
$$

where $f(t)$ is data and $\varphi$ is wavelet function. For small scales, we expect

$$
Z_{q}(a) \sim a^{\tau(q)} .
$$

First, we investigated the changes in $Z_{q}(a)$ in the time series at a different scale a for each moment $q$. We plotted the logarithm of $Z_{q}(a)$ against that of time scale a. Here $\tau(q)$ is the slope of the fitted straight line for each $q$. Next, we plotted $\tau(q)$ versus $q$, which is the multifractal spectrum. The time window was advanced by one year, which was repeated. Monofractal and multifractal signals were defined as follows: A monofractal signal corresponds to a straight line for $\tau(q)$, while a multifractal signal $\tau(q)$ is nonlinear [9]. We calculated the $R^{2}$ value, which is the coefficient of determination, for the fitted straight line. If $R^{2} \geq 0.98$, the time series is monofractal; if $0.98>R^{2}$, it is multifractal.

A time window was fixed to 6 years for the following reasons. We calculated the wavelets with time windows of 10,6 , and 4 years. Initially, when a time window was 10 years, a fractality changed slowly. By integrating the wavelet coefficient in a wide range, small changes were canceled. Thus, this case was inappropriate to find a fast change of climatic regime shift. Next, when the time window was 4 years, the fractality changed quickly. The overlap of the first and following data was 3 years, which was shorter than the 9 years when the time window was 10 years, and the change of fractality was large. Thus, this case was also inappropriate. Finally, when the time window was 6 years, a moderate change in fractality was observed. Hence, the time window was fixed to 6 years.

We calculated the multifractal spectrum $\tau(q)$ of the SSN between 1910 and 2010. The multifractal spectrum $\tau(q)$ between 1967 and 1979 is shown in Figure 1. The data were analyzed in 6-year sets; as an example, the multifractal spectrum $\tau(q)$ of s67 was calculated between 1967 and 1972. To study the change in fractality, the time window was advanced by one year and the multifractal spectrum $\pi(q)$ was obtained from s67 to s76. A monofractal signal corresponds to a straight line for $\tau(q)$, whereas for a multifractal signal, $\tau(q)$ is nonlinear. In Figure 1 , the constantly changing curvature of the $\tau(q)$ curves for $s 67, \mathrm{~s} 68$, and $\mathrm{s} 72$ s74 suggests multifractality. In contrast, $\tau(q)$ is linear for s69 - s71, which indicates monofractality.

We plotted the $\tau(-6)$ of each index, where $q=-6$ is the appropriate number for showing a change in $\tau$. The large negative values of $\tau(-6)$ show large 


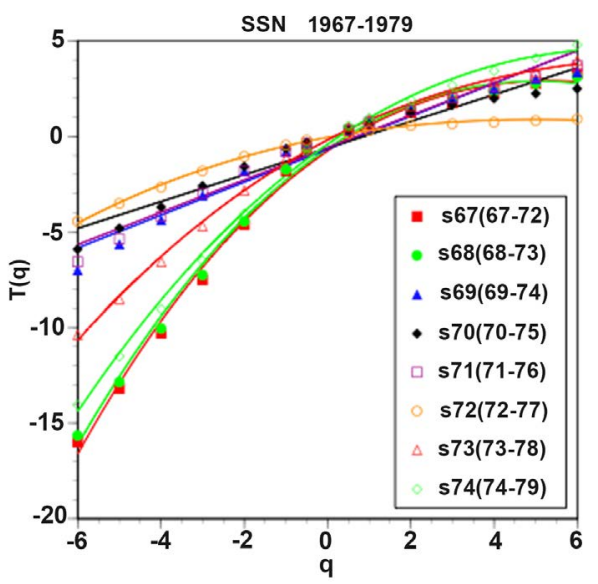

Figure 1. Multifractal spectrum $\tau(q)$ for individual SSN between 1967 and 1979.

multifractality. Thus, $\pi(-6)$ is not always equal to the fractality gained from the $\mathrm{R}^{2}$ value.

\section{Results}

We investigated the change in the SSN. To detect the changes in multifractality, we examined the multifractal analysis of the SSN. The $\tau(-6)$ of the SSN and SSN as well as the solar cycle number of the SSN are shown in Figure 2. The SSN has an 11-year and a 22-year cycle for the reversal of the Sun's magnetic field. $\tau(-6)$ is the value of $\tau$ for $q=-6$ and indicates the fractality of the index. The red square and green circle represent monofractality and multifractality for the six years around the plotted year, respectively. For example, the red square for 1962 in the SSN shows monofractality between 1959 and 1964. The data are excluded if we cannot distinguish between monofractality and multifractality.

Fractal properties were regularly changing. When the SSN was maximum (minimum), the $t(-6)$ of the SSN became maximum, as shown by 1 (3) in Figure 2, when the SSN showed monofractality or weak multifractality. The $\pi(-6)$ of the SSN became minimum two years before the maximum SSN as shown by 2 in Figure 2, when the change in the SSN was the largest as shown in Figure 2. About three years before the minimum SSN, the $\tau(-6)$ of the SSN became maximum as shown by 4 in Figure 2 and the multifractality was the weakest. Before the maximum SSN, the multifractality of the SSN became the strongest and at the maximum SSN they became the weakest, which indicated a change from multifractality to monofractality.

The solar polar field is shown in Figure 3. The changes in the solar polar field were large two years before the maximum SSN of the solar cycles 22, 23, and 24 as shown by the arrow in Figure 3, which was determined from the slope of the change in the graph in Figure 3. The solar polar field became maximum and after a while the SSN became minimum.

Next, we show the $t(-6)$ of the solar polar field and the solar polar field in Figure 4. Fractal properties were regularly changing. When the solar polar field 


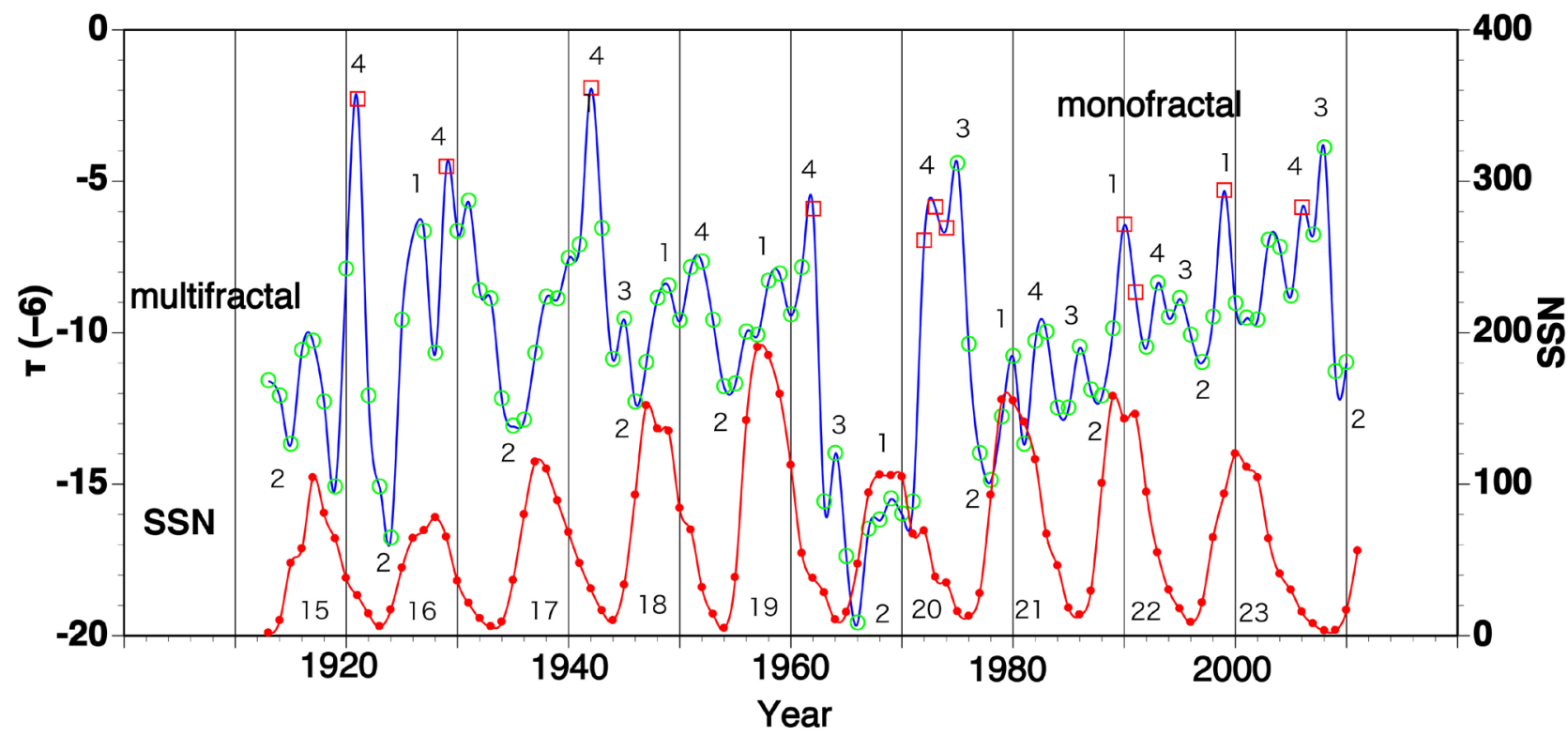

Figure 2. Time series of the $t(-6)$ of the SSN and the SSN. Cycle number of the SSN is also shown. The $\tau(-6)$ is the value of $\tau$ for $q=-6$. The red squares show monofractality and the green circles show multifractality for a 6 -year period centered on the year shown Numbers are explained in the text.

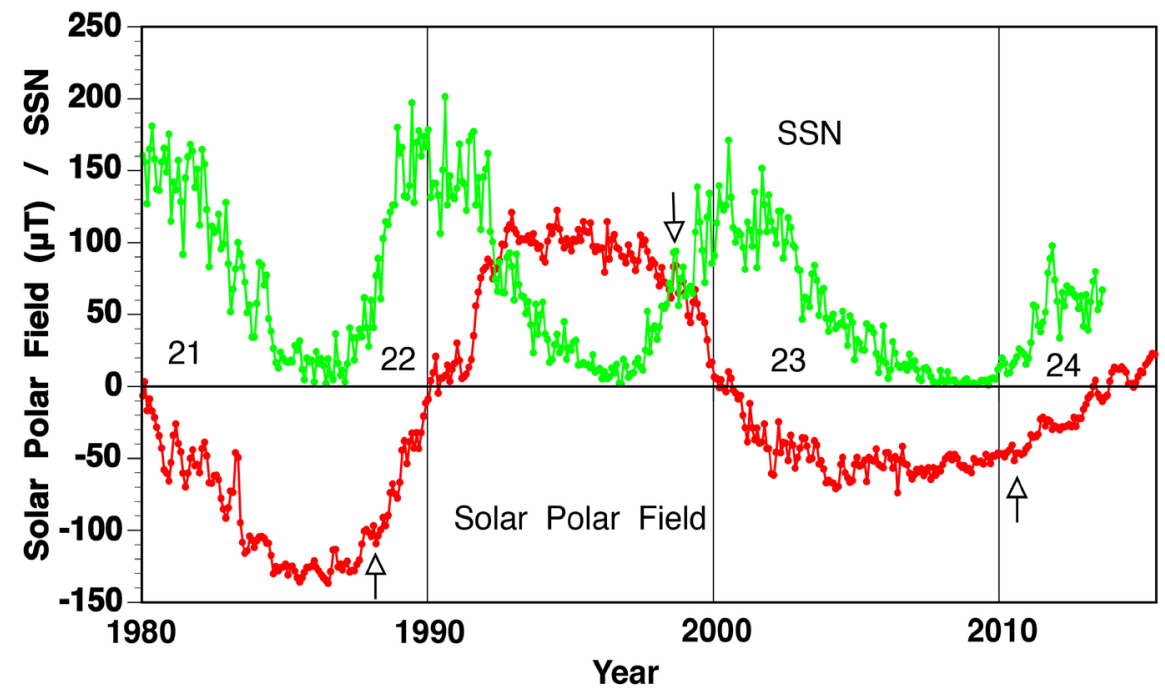

Figure 3. Time series of the solar polar field and SSN.

became maximum, the $\tau(-6)$ of the solar polar field became maximum, as shown by 5 in Figure 4, when the solar polar field indicates monofractality or weak multifractality. When the $\tau(-6)$ of the solar polar field became minimum, as shown by 6 in Figure 4, the solar polar field changed largely. The solar polar field was zero in 1991 and 2000 in Figure 4, when the fractality were monofractal and weak multifractal, respectively as shown by 7. A maximum of the solar polar field had two peaks of $\pi(-6)$ in cycles 21,22 , and 23.

We show the $\tau(-6)$ of the solar polar field and SSN between 1980 and 2015 in Figure 5. When the solar polar field was maximum, the SSN was minimum in cycles 21 and 22. After the maximum solar polar field, the SSN became 


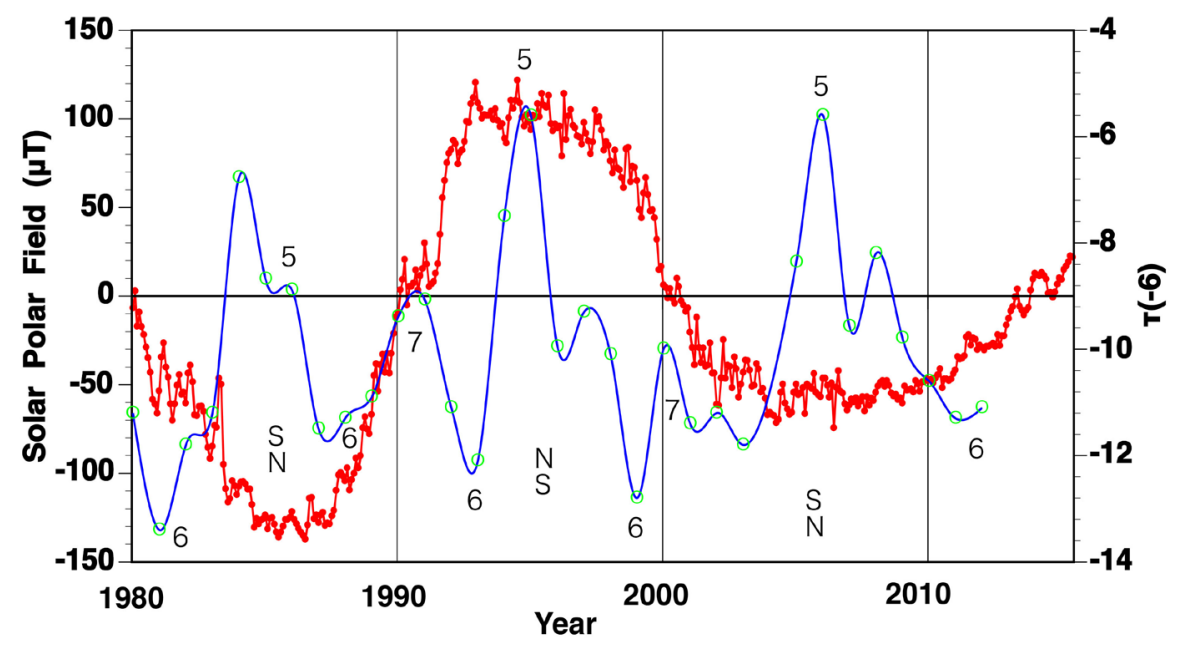

Figure 4. Time series of the $t(-6)$ of the solar polar field and the solar polar field. Numbers are explained in the text. SN indicates the magnetic pole of the solar polar field.

minimum in cycle 23. Those results coincided with the result of Figure 3 except for cycle 22. The multifractality of SSN became large before two year of SSN maximum shown by 2 in Figure 5, then that of the solar polar field became large shown by 6 and changed largely.

\section{Discussion}

When the SSN and solar polar field were maximum and minimum, the $t(-6)$ of the SSN and solar polar field strength became maximum. The fractal dimensions decrease with increasing mean magnetic field, implying that the magnetic field distribution is more regular in active regions [10]. When the solar polar field was large, the multifractality was weak, which coincides with the result of Ioshpa et al. [10]. The peaks of $t(-6)$ for the SSN are classified into four peaks, the maximum peaks at the maximum SSN, the minimum SSN, and the maximum peak at three years before the minimum SSN and the minimum peak at two years before the maximum SSN. The $\tau(-6)$ of the SSN became minimum two years before the maximum SSN of solar cycles 22, 23, and 24 . The multifractality of the SSN became strong two years before the maximum SSN, when the changes in the SSN were large. When the SSN became maximum, the fractality of the SSN changed from multifractality to monofractality. In our previous paper [11], in terms of the multifractal analysis, we conclude that a climatic regime shift corresponds to a change from multifractality to monofractality of the Pacific Decadal Oscillation (PDO) index.

When the change in the solar polar field was small, the multifractality was weak. The solar polar field showed weak multifractality when the solar polar field was maximum and minimum. We compared the $\tau(-6)$ of SSN with the solar polar field. In Figure 3, the solar polar field became maximum, and then the SSN became minimum. The solar polar field is the strongest during an interval of about three years before the sunspot minimum [12]. From the fractal property, 


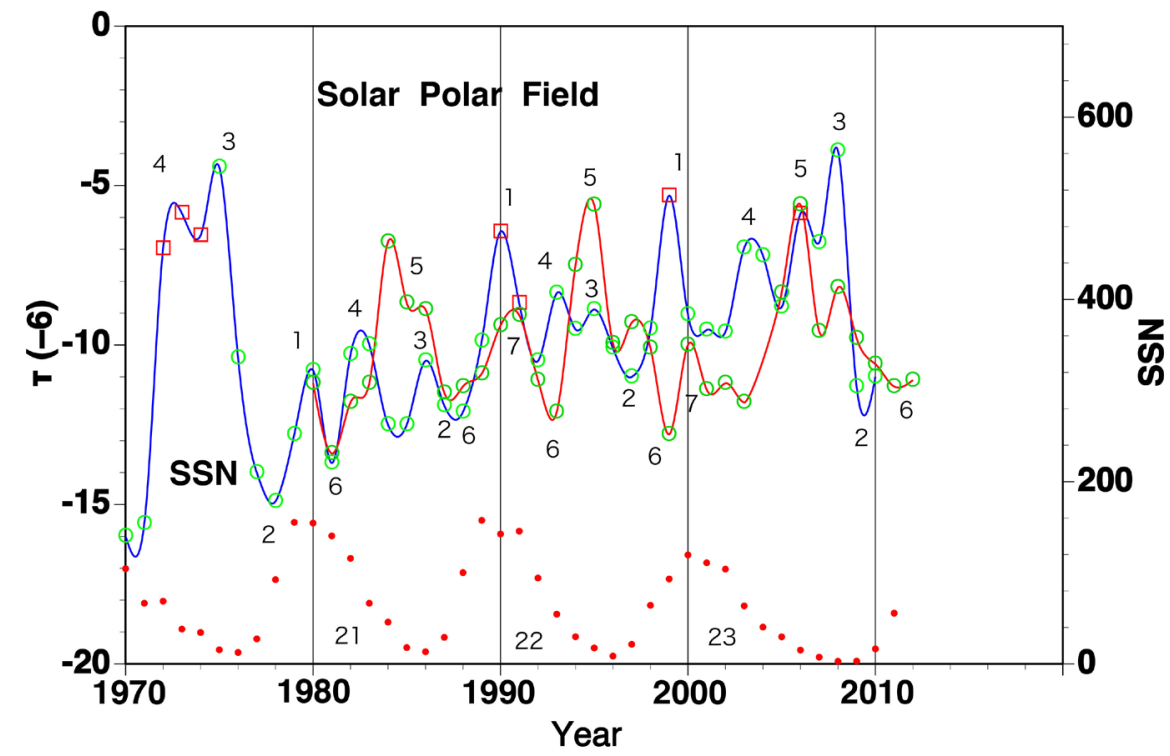

Figure 5. Time series of the $\tau(-6)$ of the average solar polar field and SSN. The red squares show monofractality and the green circles show multifractality for a 6-year period centered on the year shown. SSN and Solar cycle number of the SSN are also shown. Numbers are explained in the text.

comparing with 5 (the solar field maximum) and 3 (the SSN minimum) shown in Figure 5, at the same time in cycles 21 and 22 and there was a difference in cycle 23 . About three years before the minimum SSN, the $t(-6)$ of the SSN became maximum and the multifractality was the weakest, then the $\tau(-6)$ of the solar polar field also became maximum and the multifractality was the weakest. The multifractality of SSN became large before two year of SSN maximum, then that of the solar polar field became large and changed largely. It was found that the change in SSN triggered the change in the solar polar field referring to Figure 4 and Figure 5. Hence, the SSN and solar polar field closely correlated from the view point of fractals.

The solar polar field makes the magnetic field of the sunspots when the solar cycle is the minimum [13]. In this study, we compared the maximum SSN with the maximum solar polar field before the maximum SSN. When the maximum solar polar field before the maximum SSN was larger, the maximum SSN of the next cycle was larger. Hence, the formation of the magnetic field of the sunspots was correlated with the solar polar field.

\section{Conclusions}

In this study, we investigated the relation between the SSN and the solar polar field by performing a multifractal analysis. To detect the changes in multifractality, we performed a multifractal analysis on the SSN, and the solar polar field using the wavelet transform.

1) When the SSN was maximum and minimum, the SSN showed monofractality or weak multifractality. The solar polar field showed weak multifractality 
when that was maximum and minimum.

2) When the SSN became maximum, the fractality of the SSN changed from multifractality to monofractality.

3) The multifractality of SSN became large before two years of SSN maximum, then that of the solar polar field became large and changed largely. It was found that the change in SSN triggered the change in the solar polar field. Hence, the SSN and solar polar field were closely correlated from the view point of fractals.

4) When the maximum solar polar field before the maximum SSN was larger, the maximum SSN of the next cycle was larger. The formation of the magnetic field of the sunspots was correlated with the solar polar field.

\section{Conflicts of Interest}

The author declares no conflicts of interest regarding the publication of this paper.

\section{References}

[1] Svensson, C., Olsson, J. and Berndtsson, R. (1996) Multifractal Properties of Daily Rainfall in Two Different Climates. Water Resources Research, 32, 2463-2472. https://doi.org/10.1029/96WR01099

[2] Ivanov, P.Ch., Amaral, L.A.N., Goldberger, A.L., Havlin, S., Rosenblum, M.G., Struzikk, Z.R. and Stanley, H.E. (1999) Multifractality in Human Heartbeat Dynamics. Nature, 399, 461-465. https://doi.org/10.1038/20924

[3] Cliver, E.W., Boriakoff, V. and Feynman, J. (1998) Geomagnetic Activity and the Solar Wind during the Maunder Minimum. Geophysical Research Letters, 25, 897-900. https://doi.org/10.1029/98GL00500

[4] Cliver, E.W., Boriakoff, V. and Feynman, J. (1998) Solar Variability and Climate Change: Geomagnetic aa Index and Global Surface Temperature. Geophysical Research Letters, 25, 1035-1038. https://doi.org/10.1029/98GL00499

[5] Kane, R.P. (2002) Evolution of Geomagnetic aa Index near Sunspot Minimum. Annales Geophysicae, 20, 1519-1527. https://doi.org/10.5194/angeo-20-1519-2002

[6] Kane, R.P. (2002) Prediction of Solar Activity: Role of Long-Term Variations. Journal of Geophysical Research, 107, Article ID: 1113. https://doi.org/10.1029/2001JA000247

[7] Bucha, V. and Bucha Jr., V. (1998) Geomagnetic Forcing of Changes in Climate and in the Atmospheric Circulation. Journal of Atmospheric and Solar-Terrestrial Physics, 60, 145-169. https://doi.org/10.1016/S1364-6826(97)00119-3

[8] Muzy, J.F., Bacry, E. and Arneodo, A. (1991) Wavelets and Multifractal Formalism for Singular Signals: Application to Turbulence Data. Physical Review Letters, 67, 3515-3518. https://doi.org/10.1103/PhysRevLett.67.3515

[9] Frish, U. and Parisi, G. (1985) On the Singularity Structure of Fully Developed Turbulence. In: Ghil, M., Benzi, R. and Parisi, G., Eds., Turbulence and Predictability in Geophysical Fluid Dynamics and Climate Dynamics, North-Holland, New York, 84-88.

[10] Ioshpa, B.A., Obridko, V.N. and Rudenchik, E.A. (2008) Fractal Properties of Solar Magnetic Fields. Astronomy Letters, 34, 210-216. 
https://doi.org/10.1134/S1063773708030080

[11] Maruyama, F., Kai, K. and Morimoto, H. (2015) Wavelet-Based Multifractal Analysis on Climatic Regime Shifts. Journal of the Meteorological Society of Japan, 93, 331-341. https://doi.org/10.2151/jmsj.2015-018

[12] Svalgaard, L. and Kamide, Y. (2005) Sunspot Cycle 24: Smallest Cycle in 100 Years? Geophysical Research Letter, 32, Article ID: L01104. https://doi.org/10.1029/2004GL021664

[13] Upton, L. and Hathaway, D.H. (2014) Predicting the Sun's Polar Magnetic Fields with a Surface Flux Transport Model. The Astrophysical Journal, 780, Article ID: 5. https://doi.org/10.1088/0004-637X/780/1/5 